\title{
Trabajando con Pósteres: una Herramienta para el Desarrollo de Habilidades de Comunicación en la Educación de Pregrado
}

Tatiana Canales ${ }^{(1)}$ y Rodolfo Schmal ${ }^{(2)}$

(1) Escuela de Psicología, Facultad de Psicología, Universidad de Talca Campus Lircay, Avenida Lircay S/N, Talca-Chile (e-mail: tcanales@utalca.cl)

(2) Escuela de Ingeniería Informática Empresarial, Facultad de Ciencias Empresariales, Universidad de Talca Campus Lircay, Avenida Lircay S/N, Talca-Chile (e-mail: rschmal@utalca.cl)

Recibido Jul. 31, 2012; Aceptado Sep. 06, 2012; Versión final recibida Sep. 25, 2012

\section{Resumen}

Se describe la experiencia en aula en una carrera universitaria de pregrado sobre la presentación de trabajos en forma de pósteres o paneles, como se usan en los congresos y conferencias. Las carreras en la universidad de Talca promueven como parte de la formación el desarrollo de las competencias de trabajo en equipo y comunicacionales. Utilizando metodologías cualitativas y cuantitativas no paramétricas, se analizó y reportó el progreso académico de un grupo de estudiantes de uno de los cursos de la carrera con la práctica y presentación de sus trabajos en forma de póster. Los resultados señalan que para aproximadamente $67 \%$ de los estudiantes fue su primera experiencia de trabajo con pósteres y más del $80 \%$ de los encuestados calificó esta experiencia como positiva o muy positiva. Los estudiantes reportan que el trabajo con pósteres permite desarrollar capacidades de comunicación que el mercado profesional está demandando.

Palabras clave: pósteres, competencias, trabajo en equipo, educación de pregrado, habilidades de comunicación

\section{Working with Posters: a Tool for Fostering Communication Skills in Undergraduate Education}

\begin{abstract}
The teaching experience with a university career about the use of poster for presenting a defined work, similar to what is used in congress and conferences. The careers at the University of Talca promote workgroup and communicational skills as part of the formation of the students. Thus, using qualitative and non-parametric quantitative analyses, the academic progress during the preparation and presentation of the posters of a group of students was analyzed. Results show that more than about $67 \%$ of the group reports that it was their first time using posters in their academic training and more than $80 \%$ of the students valued this experience as positive or very positive. Finally, students report that this kind of work allows them to develop communication skills that are highly needed in the professional market.
\end{abstract}

Keywords: posters, competences, teamwork, undergraduate education, communication skills. 


\section{INTRODUCCION}

El objetivo del presente trabajo es dar a conocer la experiencia de presentación de pósteres por parte de estudiantes en un par de asignaturas de una carrera de pregrado, de Ingeniería en Informática Empresarial, que se imparte en la Universidad de Talca (Chile). Ésta es una universidad estatal chilena que surge en 1981 en la ciudad de Talca, capital de la región del Maule y a $250 \mathrm{~km}$ al sur de Santiago de Chile, que se ha propuesto formar profesionales que se distingan por una formación basada en competencias y bajo un marco valórico que busca la excelencia, la innovación tecnológica, y el compromiso con el progreso y bienestar regional y del país, en permanente diálogo e interacción con el entorno social, cultural y económico, tanto local como global (UTalca, 2012). Actualmente cuenta con una matrícula del orden de 7,000 estudiantes de los cuales alrededor de 6,000 son de las 20 carreras de pregrado que se imparten. Esta matrícula es atendida por una planta académica de 380 académicos, de los cuales sobre el $80 \%$ tiene estudios de postgrado (magísteres y doctorados).

Para apoyar la implementación de un nuevo modelo educativo basado en el desarrollo de competencias se ha creado una Vicerrectoría de Docencia de Pregrado, y dentro de ella, el Centro de Innovación y Calidad Docente (CICAD) cuya propósito fundamental es acompañar a los académicos en el proceso de rediseño curricular asociado a un cambio que afecta a los académicos docentes, sus esquemas evaluativos, metodologías de trabajo en aula, su relación con las empresas, así como su relación con los estudiantes al involucrarse en sus procesos de aprendizaje.

En este marco, en el año 2005 surge en la Facultad de Ciencias Empresariales de la Universidad de Talca un programa de pregrado conducente al título de Ingeniero en Informática Empresarial (IIE), destinado a satisfacer la necesidad del mercado de disponer con ingenieros con una formación informática y de gestión que les permita desenvolverse en mercados crecientemente digitales y asumir posiciones de responsabilidad en los ámbitos: a) del desarrollo de sistemas informáticos; b) de la gestión de proyectos informáticos; c) de la elaboración de planes informáticos alineados con la estrategia del negocios; y d) de la dirección de organizaciones informáticas y de la negociación o interacción con ellas (Schmal y Ruiz-Tagle, 2009).

Dentro de los principios orientadores centrales presentes en la creación de esta carrera enfocada al desarrollo de competencias se encuentra (Schmal y Ruiz-Tagle, 2011):

a) Aprender haciendo (Learning by doing), en consideración al perfil del alumnado que se caracteriza por tener un estilo de aprendizaje activo en base a experiencias concretas ya que al hacerlo logran plasmar su aprendizaje en una situación real motivados por su interés en vivir las experiencias, experimentar, sentir, generar ideas sin limitaciones formales o de estructura donde el protagonista del proceso enseñanzaaprendizaje es el alumno y no el profesor (Venables et al., 2009).

b) Implementar la enseñanza dual mediante una fuerte vinculación con empresas a través del desarrollo de proyectos reales asociados a la resolución de problemas que enfrentan las empresas mediante la implementación de talleres y prácticas en modalidad de consultoría (Greinert, 2004; Araya, 2008). El espíritu que inspira este principio es el que se observa en las carreras de la salud cuyo objeto de estudio son los pacientes, por lo que es habitual que en el proceso de enseñanza los alumnos deban cursar asignaturas denominadas "clínicas" donde aplican sus conocimientos en pacientes bajo la supervisión de un docente. En la carrera el sujeto de estudio son las empresas, en las cuales los alumnos deben realizar proyectos que apuntan a resolver problemas o desafíos que ellas encaran.

c)Aplicar la innovación como concepto que atraviese toda la carrera, lo que se expresa en la frase "formando para innovar", y que implica alentar nuevas iniciativas, nuevas miradas, nuevas formas de hacer las cosas. Desde su concepción esta carrera responde a una necesidad de satisfacer la brecha entre la demanda de soluciones de gestión y la oferta de TIC's, que se manifiesta en una alta demanda de profesionales que puedan integrar estos dos mundos.

d) Valorar el aprendizaje de los alumnos en distintos contextos -empresas, otras carreras o universidadesalentando por tanto la movilidad estudiantil y la existencia de módulos electivos que recojan los cambios producidos en los mercados laboral y tecnológico. Esto, en atención al perfil de los estudiantes, provenientes en su mayoría de familias cuya situación económica no les ha permitido conocer otros países ni otras culturas por lo que la movilidad estudiantil puede ser un importante aporte en su formación al abrir sus horizontes y alimentarse de las realidades de alumnos, profesores y ciudadanos de otras latitudes, lo que se traduce en el desarrollo de visiones holísticas del entorno (González et al., 2004; Brunner, 2008; Prieto et al., 2011). 
e) Desarrollar competencias transversales vinculadas con el desarrollo personal, la formación ciudadana, el pensamiento analítico y las habilidades de comunicación oral y escrita. Este interés se explica porque ellas no se encuentran presentes dentro de los niveles esperados en los alumnos matriculados y que se consideran imprescindibles para ingresar a un mercado laboral que valora crecientemente estas competencias (Schmal, 2012).

Lo anterior ha implicado el rediseño del plan de formación de la carrera bajo este nuevo paradigma y por tanto, profundos desafíos a todo nivel, entre los que se destacan (Cates y Langford, 1999): a) el desarrollo de competencias de comunicación; y b) el fortalecimiento de la vinculación con la sociedad y las empresas.

El primero, porque en tan solo tres décadas, la cobertura de la educación superior en Chile que estaba por debajo del $20 \%$, actualmente está bordeando el $40 \%$. Por consiguiente, sus universidades, en particular las universidades públicas, necesariamente deben hacerse cargo de la incorporación de un alumnado cuyas familias poseen bajo capital sociocultural, y son de primera generación, esto es, donde los padres no son profesionales (Brunner, 2007). Estos alumnos, por el ambiente en que se han desenvuelto tienden a presentar déficits de competencias de comunicación, tanto orales, como escritas, imprescindibles para desenvolverse con éxito en mercados laborales cada vez más exigentes.

El segundo desafío, estrechar vínculos con la sociedad y las empresas, se aborda con un modelo curricular orientado al desarrollo de competencias, el cual no se entiende si no se asocia con la educación dual o la educación cooperativa, con un activo protagonismo de la sociedad y las empresas en el proceso de enseñanza y aprendizaje. La empresa y la sociedad son los espacios donde la competencia, tal como se tiende a concebir actualmente, pone su énfasis en su puesta en acción.

\section{COMPETENCIAS}

En Europa, el concepto de competencia cobra fuerza al amparo del proceso de Bolonia, definido como un proceso destinado a establecer un espacio europeo de educación superior que en lo esencial facilite la movilidad de los trabajadores por la vía del reconocimiento de titulaciones, el establecimiento de equivalencias y sistemas de transferencias entre distintas universidades (Brunner, 2008). Bajo este contexto se ha estado tendiendo a un diseño curricular universitario orientado al desarrollo de competencias, entendiéndose la competencia como "una combinación dinámica de atributos, en relación a conocimientos, habilidades, actitudes y responsabilidades, que describen los resultados del aprendizaje de un programa educativo o lo que los alumnos son capaces de demostrar al final de un proceso educativo" (Bezanilla, 2003).

Si bien el concepto proviene del mundo laboral y tiene una fuerte connotación utilitaria, una competencia envuelve la demostración de la capacidad para un desempeño satisfactorio no solo en ámbitos definidos dentro de las organizaciones, sino que va más allá de ellas, en la sociedad en que nos desenvolvemos (Aristimuño, 2004). De allí que se afirme que la competencia es la puesta en acción, movilización, despliegue e integración de conocimientos, habilidades y destrezas, lo que implica que un diseño curricular orientado al desarrollo de competencias debe estar guiado en lo sustancial por estrategias que destaquen la relevancia y pertinencia de los contenidos que se aprenden; eviten el fraccionamiento tradicional de los conocimientos y faciliten su integración; generen aprendizajes en situaciones complejas; y por último, estimulen, faciliten y provoquen la autonomía personal del estudiante.

Siguiendo a Le Boterf (2001), en la Universidad de Talca, el proceso de formación de un profesional competente implica formar un profesional capaz de "saber actuar en un contexto particular, poniendo en juego los recursos personales y contextuales (incluyendo redes) para la solución de un problema específico, con un proceso de reflexión sobre lo que se está haciendo" (Faúndez et al., 2009). Esta interacción es implementada a través de una estrecha vinculación de la Escuela de Ingeniería en Informática Empresarial con una red de empresas en las cuales se aspira que los futuros profesionales se desempeñen. Para estos efectos las empresas asociadas a la red están cumpliendo el rol de laboratorios, proporcionando un ambiente de aprendizaje vía resolución de casos, realización de prácticas, y desarrollo de proyectos (Schmal et al., 2009).

\section{PÓSTERES}

Los métodos contemporáneos de educación superior están dirigidos a promover individuos con alto nivel de conocimiento, pero también con habilidades de resolución de problemas y de aprendizaje auténtico, como la capacidad de autorregulación, comunicación y cooperación con otros. Los estudiantes que luego trabajarán en organizaciones contemporáneas necesitan poder analizar información para mejorar sus habilidades de toma de decisiones y comunicativas, además de tomar una posición reflexiva en su proceso de aprendizaje. 
En una docencia consecuente, por lo tanto, la evaluación de tales habilidades debe ir más allá de la medición de la capacidad de reproducción del conocimiento (Dochy et al., 1999).

El uso de pósteres como herramienta didáctica ha sido sugerido como una forma de promover habilidades relevantes en la formación profesional de los estudiantes, como por ejemplo: el manejo de recursos bibliográficos, capacidad de seleccionar material, pensamiento crítico, capacidad de síntesis, trabajo cooperativo y habilidades de presentación (Bracher et al., 1998). Facilita que los estudiantes integren conceptos y demuestren su comprensión al compartir su trabajo con pares y docentes (Handron, 1994). El aspecto social configura una de las mayores ventajas de este método, en cuanto genera una posibilidad muy potente de generación de redes a partir de la obtención de retroalimentación desde una variedad de fuentes, entre ellas sus pares, docentes, otros profesionales y potenciales usuarios o clientes (Moneyham et al., 1996), además de ser una plataforma de difusión de resultados y de innovaciones muy rápida (Moore et al., 2001). El entusiasmo que se genera es contagioso entre estudiantes y docentes, y tiene altas probabilidades de enriquecer el ambiente de aprendizaje mas allá de los límites de la sala de clases (Handron, 1994).

El uso de pósteres no es un recurso especialmente nuevo. Ya en 1980 se ha descrito en la promoción de aprendizajes, desarrollo de habilidades comunicativas y de generación de redes, además de las capacidades metacognitivas de pensamiento crítico y síntesis de información. Además, se presenta como una forma novedosa de evaluación de la comprensión del estudiante (Bracher et al., 1998) en cuanto facilita que los estudiantes relacionen el conocimiento teórico en un proceso de cuestionamiento, que demuestra su nivel de comprensión (Handron, 1994). Por otra parte, los pósteres se pueden adaptar para varios tipos de aprendizajes, ya sea representando una línea de pensamiento, un caso clínico, una investigación, una propuesta, etc. La actividad en si misma requiere de más tiempo de preparación, que otros formatos evaluativos o de aprendizaje, pero se presenta como un medio justo, inclusivo y eficiente para los docentes en su calificación (Handron, 1994), lo que lo hace una opción muy positiva de incorporar a la formación de profesionales.

La función del/a docente en este método es de estratega, consultor/a y facilitador/a, siendo este último el más importante cuando los estudiantes dialogan y critican los distintos trabajos o temáticas trabajadas (Bracher et al., 1998). En un póster, los estudiantes pueden asumir el rol de asesores para generar una multiplicidad de puntos de vista en auto, co y heteroevaluación de sus logros (Dochy et al., 1999). Esta evaluación por pares y autoevaluación genera la posibilidad que ambos definan los resultados esperables de aprendizaje de la tarea asignada, que se dialogue significativamente entre estamentos para definir estándares de calidad del trabajo y, por último, que se genere una evaluación del proceso que lleva a la construcción del póster en vez de sólo el producto (Orsmond et al., 2000).

Reportes de actividades de generación y presentación de pósteres en educación superior en el área de la salud han descrito una reacción positiva de los estudiantes a la actividad, donde los estudiantes reconocen que han podido utilizar su conocimiento y desarrollar un sentido de logro en la apropiación de su trabajo (Bracher et. Al., 1998) ya que los pósteres les dan la oportunidad de ser creativos/as y autodirigidos/as en la generación de un producto único (Handron, 1994). Sin embargo, poca información se ha reportado en otras áreas de formación y currículo específicos, por lo que una experiencia de desarrollo de pósteres en cursos cuya metodología de aprendizaje esté basada en proyectos reales desarrollados en empresas, en el marco de una formación orientada al desarrollo de competencias es especialmente atractivo de documentar y discutir (Lester y Costley, 2010).

\section{LA EXPERIENCIA}

Los planes de estudios de los estudiantes de las carreras de pregrado en la Universidad de Talca (Chile) contemplan un componente de formación fundamental destinada a desarrollar competencias genéricas, entre las que destacan las comunicacionales y de trabajo en equipo. Este componente es llevado a cabo en los dos primeros años a fin de que las competencias implicadas sean puestas en acción en los cursos responsables de la formación disciplinar.

Para estos efectos, en dos asignaturas de la carrera de Ingeniería en Informática Empresarial de la Facultad de Ciencias Empresariales de la Universidad de Talca (Chile) se está llevando a cabo la metodología de aprendizaje basado en proyectos que deben realizarse en empresas. Entre los entregables exigidos a los alumnos se incluyen fichas de las empresas, presentaciones orales del proyecto y exposición de pósteres. Estos últimos son concebidos como un medio estático y visual destinado a comunicar las ideas y mensajes centrales de un proyecto, capaces de ser transmitidas por los alumnos sin necesidad de explayarse en una narrativa oral. Durante la exposición de un póster, sus responsables tan solo deben estar disponibles para 
aclarar dudas, responder consultas a los interesados, atraer y entusiasmar a potenciales destinatarios, y eventualmente precisar detalles a petición de éstos.

En la elaboración de todo póster se deben considerar tanto aspectos de forma como de fondo. Respecto de estos últimos, se exigió: a) El título del proyecto, el cual debe ser sugerente, expresivo, acompañado de los nombres de los autores y su afiliación; b) Una descripción sintética del tema, problema o proyecto que se ha abordado: lo que se aspira resolver, descubrir, evidenciar o establecer, sugiriendo el objetivo a alcanzar; c) Una justificación del tema, en el que se expliquen los fundamentos por los cuales se está abordando; d) La metodología bajo la cual se ha enfrentado el tema; e) Los resultados alcanzados junto con los hallazgos más importantes encontrados; y f) Las limitaciones bajo las cuales se desarrolló el trabajo, acompañadas con las recomendaciones a seguir que el trabajo realizado aconseja. En cuanto a los aspectos de forma, su presentación debe ser capaz de atraer, concitar interés, de fácil lectura e interpretación, razón por la cual debe distribuirse su contenido en una secuencia que facilite su lectura y la apropiación de la idea, del concepto o mensaje que se aspira transmitir. Considerando la máxima de que una imagen dice más que mil palabras, suelen incluir gráficas, tablas, figuras.

El aprendizaje basado en proyectos está fuertemente asociado al aprendizaje colaborativo, esquema que se apoya en el trabajo en grupo por parte de los alumnos (Johnson et al., 1998). La docencia de las asignaturas en las que se exigió la exposición de pósteres, incluyó la supervisión en el desarrollo de proyecto y el desarrollo gradual de las competencias esperadas a partir de un trabajo cooperativo presencial y no presencial, donde los estudiantes son responsables tanto del producto grupal, como de sus funciones específicas en la dinámica de trabajo. Los estudiantes no solo tienen que ser capaces de llevar a buen término sus respectivos proyectos, esto es, que tengan como resultados productos/servicios en base a los requerimientos, sino que también deben ser apropiadamente comunicados. En tal sentido, la presentación de los resultados del proyecto mediante la exposición de pósteres constituye una interesante opción complementaria a aquellas basadas en presentaciones y/o informes ejecutivos u otros.

La iniciativa nació en el año 2006 en la cátedra de Psicología de la Educación de la carrera de Psicología de la Universidad de Talca con alumnos del último año como una forma de traer a nivel de la formación de pregrado lo que es una práctica habitual en congresos científicos, como es la presentación de resultados de proyectos de investigación vía pósteres. En esta experiencia llamó poderosamente la atención el entusiasmo y la seriedad con que fue asumida la responsabilidad por parte de los distintos grupos de trabajo. Posteriormente esta idea, con variantes, ha estado siendo replicada desde el año 2008 (con excepción del año 2011 con motivo de las movilizaciones estudiantiles) en dos asignaturas de la carrera de Ingeniería en Informática Empresarial de la misma universidad, aprovechando que ambas exigen el desarrollo de proyectos por parte de grupos de alumnos. Estas asignaturas fueron:

Análisis y Diseño Lógico de Sistemas de Información (8 ECTS) incluido en el segundo año con el propósito que los alumnos tengan las competencias que le permitan capturar los requerimientos funcionales de información ante una problemática dada en una empresa, y modelar los datos y procesos que permitan satisfacer los requerimientos recogidos; y

Modelamiento de Negocios y Rediseño Organizacional (8 ECTS) asignatura que se encuentra localizada en el cuarto año del plan de formación con el propósito de que los alumnos sean capaces de modelar un negocio ya existente a partir de su misión, visión, objetivos, estrategias, proyectos y procesos, para posteriormente identificar el diseño organizacional más apropiado para la implementación exitosa y eficiente del modelo de negocios determinado.

En ambas asignaturas se exigen los siguientes entregables asociados a los proyectos que realicen los alumnos: Elaboración de ficha: que incluye información de identificación del grupo de trabajo, de la empresa, y del problema a resolver; Elaboración de informe ejecutivo: documento que incluya un resumen de no más de 200 palabras, una introducción, un desarrollo, resultados, conclusiones y bibliografía; Presentación oral: exposición de no más de 15 minutos en diapositivas, ante una audiencia compuesta por los alumnos y profesores de la asignatura y los representantes de las empresas en las cuales fueron realizados los proyectos, seguido por 15 minutos de consultas y debate en torno a los resultados alcanzados; y Exposición de póster: despliegue de póster físico en las paredes de la sala de clases para ser visto, leído, analizado, discutido y evaluado por alumnos, profesores y representantes de las empresas en las cuales se han estado desarrollando los proyectos.

El formato de presentación de pósteres permitió expresar muy sintéticamente los resultados del proyecto, además de ciertos hitos relevantes de su desarrollo, que han de ser comunicados efectivamente para poder someterse a evaluación por parte de docentes de la cátedra, otros docentes, potenciales clientes y pares. El 
lenguaje y presentación debe ser un equilibrio entre el lenguaje profesional y lenguaje de difusión, para poder ser efectivo en todos los niveles ya mencionados.

La primera experiencia de exposición de pósteres permitió visualizar su atractivo para los alumnos al incentivar el trabajo colectivo y particularmente porque obliga a desarrollar una capacidad de síntesis y la presentación gráfica de los resultados logrados. Sin embargo, por realizarse la experiencia al finalizar el período académico, no hubo oportunidad de retroalimentación. Por ello, para las siguientes experiencias se han contemplado 2 exposiciones de pósteres en cada asignatura. El primer póster, llamado póster preliminar, está sujeto a una evaluación de carácter formativo con el fin de propiciar su mejoramiento. Las observaciones se volcaban a pequeñas notas autoadhesivas (post it) que eran adosadas a los pósteres correspondientes por parte de los alumnos de los diferentes grupos. Al finalizar la jornada expositiva cada grupo recogía sus respectivos pósteres con las observaciones recibidas en las notas autoadhesivas. Éstas eran complementadas por una coevaluación de cada grupo a todos los pósteres, efectuada a partir de un ordenamiento de mejor a peor póster por cada uno de los atributos dados a conocer por los profesores de las respectivas asignaturas.

Para los efectos de la evaluación, por cada póster en cada una de las asignaturas, se elaboró una plantilla de evaluación que incluía los atributos (rúbricas) a considerar, entre las que se incluyen aquellos de carácter general, tales como su legilibilidad (se lee bien?), su orden (se ve ordenado?), su limpieza (se ve despejado, sin accesorios?), su claridad (se entiende?), su completitud (está completo?); y aquellos de carácter específico, propios de la asignatura y vinculados al contenido propiamente tal del póster, y que en el caso de la asignatura de Análisis y Diseño Lógico de Sistemas de Información, incluía aspectos relativos a la descripción del negocio (incluye todos los componentes?), al diagrama y la estructura de los datos (el esquema conceptual construido captura la realidad?), a la identificación del problema (el problema se encuentra adecuadamente identificado?) y la especificación de los requerimientos (los requerimientos de información están correctamente especificados?).

Esta plantilla es tanto del profesor como de los alumnos quienes deben completar las plantillas de evaluación correspondientes a los pósteres de todos los grupos, con excepción de su propio grupo. En ambas asignaturas, a cada grupo de proyecto, conformado por 2 a 3 alumnos, se le asignó una empresa para desarrollar su respectivo proyecto.

Complementariamente, a los 34 estudiantes inscritos el año 2010 en el módulo Análisis y Diseño Lógico de Sistemas, se aplicó una encuesta al finalizar el módulo para recoger sus opiniones respecto de la experiencia desarrollada. En este módulo se trataba de identificar un problema dentro de una empresa susceptible de ser resuelto mediante un sistema de información, en cuyo caso debía identificarse el modelo de datos y de procesos que habrían de satisfacer los requerimientos de información de los usuarios del sistema. Entre las empresas en que se desarrollaron los diferentes proyectos se encuentran, entre otras, empresas del sector gastronómico, reparación de automóviles, servicios de salud, compra-venta de propiedades (inmobiliarias), capacitación educacional, gimnasios.

\section{RESULTADOS}

La evaluación de los pósteres fue realizada en base a la siguiente escala: MM (muy mejorable); $M$ (mejorable); A (adecuado); B (bien); MB (muy bien). Importa destacar que se realizaron 4 exposiciones de pósteres, lo que permitió tener hasta 4 versiones de un mismo póster. La primera versión no tuvo una evaluación formal, sirviendo tan solo de orientación para que el profesor de la asignatura hiciera hincapié en las debilidades y fortalezas encontradas. Las dos versiones siguientes tuvieron un carácter formativo, y solo la evaluación de la cuarta versión del póster fue de carácter sumativa. A modo de ejemplo, en la Tabla 1 se presenta el resultado de las evaluaciones efectuadas por los alumnos del módulo de Análisis y Diseño Lógico de Sistemas del año 2010 sobre la segunda versión del póster de uno de los grupos.

Para el cálculo de la media se asoció la calificación 1 a una evaluación MM; 2 a una evaluación M; 3 a una evaluación A; 4 a una evaluación $B$; y 5 a una evaluación MB. El resultado final se vinculó a un rango en base donde un valor bajo 2,6 se evaluó como M; entre 2,6 y 3,5, como A; entre 3,6 y 4,5, como B; y sobre 4,5, como MB.

En la Tabla 2 se muestran los resultados obtenidos sobre los 15 pósteres expuestos en sus versiones segunda, tercera y cuarta, observándose en general un progresivo avance. En la misma tabla una suerte ranking de cada póster en su versión definitiva en el que se refleja la posición relativa. El "mejor" póster sería el que se encuentra en la posición 1, y en el ejemplo corresponde al póster del grupo 5. 
Tabla 1: Evaluación del póster 1 en su segunda versión

\begin{tabular}{|l|c|c|c|c|c|c|c|}
\hline \multirow{2}{*}{$\begin{array}{c}\text { Atributo } \\
\text { (Rúbrica) }\end{array}$} & 1 & 2 & 3 & 4 & 5 & \multirow{2}{*}{ Media } & Rango \\
\cline { 2 - 7 } & MM & M & A & B & MB & & B \\
\hline Legilibilidad (se lee bien?) & 0 & 2 & 11 & 18 & 11 & 3,9 & B \\
\hline Orden (se ve ordenado?) & 0 & 0 & 8 & 22 & 12 & 4,1 & B \\
\hline Limpieza (se ve despejado?) & 0 & 0 & 8 & 17 & 17 & 4,2 & B \\
\hline Claridad (se entiende?) & 0 & 2 & 6 & 28 & 6 & 3,9 & B \\
\hline Completitud (está completo?) & 0 & 2 & 11 & 20 & 9 & 3,9 & B \\
\hline Resumen & 0 & 2 & 10 & 21 & 9 & 3,9 & B \\
\hline Conclusiones & 0 & 2 & 6 & 24 & 10 & 4,0 & A \\
\hline Diagrama & 0 & 7 & 5 & 22 & 8 & 3,7 & A \\
\hline Estructura & 0 & 5 & 11 & 20 & 6 & 3,6 & B \\
\hline Descripción del negocio & 0 & 1 & 10 & 22 & 9 & 3,9 & B \\
\hline Identificación del problema & 0 & 1 & 9 & 27 & 5 & 3,9 & A \\
\hline Especificación de requerimientos & 0 & 5 & 9 & 21 & 7 & 3,7 & B \\
\hline
\end{tabular}

Tabla 2: Evaluación de todos los pósteres

\begin{tabular}{|l|c|c|c|c|c|c|c|c|c|c|c|c|c|c|c|}
\hline \multicolumn{1}{|c|}{ PÓsTERES } & 1 & 2 & 3 & 4 & 5 & 6 & 7 & 8 & 9 & 10 & 11 & 12 & 13 & 14 & 15 \\
\hline $\begin{array}{l}\text { Legilibilidad (se } \\
\text { lee bien?) }\end{array}$ & $\mathrm{B}$ & $\mathrm{B}$ & $\mathrm{A}$ & $\mathrm{B}$ & $\mathrm{B}$ & $\mathrm{B}$ & $\mathrm{A}$ & $\mathrm{B}$ & $\mathrm{A}$ & $\mathrm{B}$ & $\mathrm{A}$ & $\mathrm{A}$ & $\mathrm{A}$ & $\mathrm{B}$ & $\mathrm{A}$ \\
\hline $\begin{array}{l}\text { Orden (se ve } \\
\text { ordenado?) }\end{array}$ & $\mathrm{B}$ & $\mathrm{A}$ & $\mathrm{B}$ & $\mathrm{B}$ & $\mathrm{B}$ & $\mathrm{B}$ & $\mathrm{B}$ & $\mathrm{B}$ & $\mathrm{B}$ & $\mathrm{B}$ & $\mathrm{A}$ & $\mathrm{A}$ & $\mathrm{A}$ & $\mathrm{B}$ & $\mathrm{A}$ \\
\hline $\begin{array}{l}\text { Limpieza (se ve } \\
\text { despejado?) }\end{array}$ & $\mathrm{B}$ & $\mathrm{B}$ & $\mathrm{B}$ & $\mathrm{B}$ & $\mathrm{B}$ & $\mathrm{B}$ & $\mathrm{B}$ & $\mathrm{B}$ & $\mathrm{B}$ & $\mathrm{B}$ & $\mathrm{A}$ & $\mathrm{A}$ & $\mathrm{A}$ & $\mathrm{B}$ & $\mathrm{A}$ \\
\hline $\begin{array}{l}\text { Claridad (se } \\
\text { entiende?) }\end{array}$ & $\mathrm{B}$ & $\mathrm{B}$ & $\mathrm{A}$ & $\mathrm{B}$ & $\mathrm{B}$ & $\mathrm{B}$ & $\mathrm{B}$ & $\mathrm{B}$ & $\mathrm{A}$ & $\mathrm{B}$ & $\mathrm{A}$ & $\mathrm{A}$ & $\mathrm{A}$ & $\mathrm{B}$ & $\mathrm{A}$ \\
\hline $\begin{array}{l}\text { Completitud } \\
\text { (está completo?) }\end{array}$ & $\mathrm{B}$ & $\mathrm{A}$ & $\mathrm{A}$ & $\mathrm{B}$ & $\mathrm{B}$ & $\mathrm{A}$ & $\mathrm{B}$ & $\mathrm{B}$ & $\mathrm{A}$ & $\mathrm{B}$ & $\mathrm{B}$ & $\mathrm{A}$ & $\mathrm{B}$ & $\mathrm{B}$ & $\mathrm{A}$ \\
\hline Resumen & $\mathrm{B}$ & $\mathrm{A}$ & $\mathrm{B}$ & $\mathrm{A}$ & $\mathrm{B}$ & $\mathrm{B}$ & $\mathrm{A}$ & $\mathrm{B}$ & $\mathrm{A}$ & $\mathrm{B}$ & $\mathrm{B}$ & $\mathrm{A}$ & $\mathrm{A}$ & $\mathrm{A}$ & $\mathrm{A}$ \\
\hline Conclusiones & $\mathrm{B}$ & $\mathrm{B}$ & $\mathrm{B}$ & $\mathrm{A}$ & $\mathrm{B}$ & $\mathrm{B}$ & $\mathrm{B}$ & $\mathrm{B}$ & $\mathrm{A}$ & $\mathrm{B}$ & $\mathrm{B}$ & $\mathrm{A}$ & $\mathrm{B}$ & $\mathrm{A}$ & $\mathrm{A}$ \\
\hline Diagrama & $\mathrm{A}$ & $\mathrm{B}$ & $\mathrm{A}$ & $\mathrm{A}$ & $\mathrm{B}$ & $\mathrm{A}$ & $\mathrm{A}$ & $\mathrm{B}$ & $\mathrm{B}$ & $\mathrm{B}$ & $\mathrm{B}$ & $\mathrm{A}$ & $\mathrm{B}$ & $\mathrm{B}$ & $\mathrm{A}$ \\
\hline Estructura & $\mathrm{A}$ & $\mathrm{A}$ & $\mathrm{A}$ & $\mathrm{A}$ & $\mathrm{B}$ & $\mathrm{A}$ & $\mathrm{A}$ & $\mathrm{B}$ & $\mathrm{A}$ & $\mathrm{B}$ & $\mathrm{A}$ & $\mathrm{A}$ & $\mathrm{B}$ & $\mathrm{A}$ & $\mathrm{B}$ \\
\hline $\begin{array}{l}\text { Descripción del } \\
\text { negocio }\end{array}$ & $\mathrm{B}$ & $\mathrm{A}$ & $\mathrm{B}$ & $\mathrm{B}$ & $\mathrm{B}$ & $\mathrm{B}$ & $\mathrm{B}$ & $\mathrm{B}$ & $\mathrm{B}$ & $\mathrm{B}$ & $\mathrm{B}$ & $\mathrm{A}$ & $\mathrm{A}$ & $\mathrm{B}$ & $\mathrm{A}$ \\
\hline $\begin{array}{l}\text { Identificación del } \\
\text { problema }\end{array}$ & $\mathrm{B}$ & $\mathrm{B}$ & $\mathrm{B}$ & $\mathrm{B}$ & $\mathrm{B}$ & $\mathrm{B}$ & $\mathrm{B}$ & $\mathrm{B}$ & $\mathrm{B}$ & $\mathrm{B}$ & $\mathrm{A}$ & $\mathrm{A}$ & $\mathrm{B}$ & $\mathrm{B}$ & $\mathrm{B}$ \\
\hline $\begin{array}{l}\text { Especificación } \\
\text { requerimientos }\end{array}$ & $\mathrm{A}$ & $\mathrm{B}$ & $\mathrm{B}$ & $\mathrm{B}$ & $\mathrm{B}$ & $\mathrm{B}$ & $\mathrm{B}$ & $\mathrm{B}$ & $\mathrm{B}$ & $\mathrm{B}$ & $\mathrm{B}$ & $\mathrm{A}$ & $\mathrm{B}$ & $\mathrm{B}$ & $\mathrm{B}$ \\
\hline \multicolumn{1}{|c|}{ Promedio } & $\mathrm{B}$ & $\mathrm{B}$ & $\mathrm{A}$ & $\mathrm{B}$ & $\mathrm{B}$ & $\mathrm{B}$ & $\mathrm{B}$ & $\mathrm{B}$ & $\mathrm{A}$ & $\mathrm{B}$ & $\mathrm{B}$ & $\mathrm{A}$ & $\mathrm{A}$ & $\mathrm{B}$ & $\mathrm{A}$ \\
\hline P2 & 3,9 & 3,8 & 3,6 & 4,2 & 4,1 & 3,7 & 3,5 & 3,8 & 3,3 & 4,1 & 3,5 & 3,5 & 2,8 & 3,0 & 2,9 \\
\hline P3 & 3,9 & 3,8 & 3,7 & 3,9 & 4,2 & 4,0 & 3,9 & 4,0 & 3,8 & 4,1 & 3,8 & 3,3 & 3,8 & 3,9 & 3,5 \\
\hline P4 & 4,3 & 4,2 & 4,1 & 4,3 & 4,5 & 4,3 & 4,1 & 4,1 & 3,5 & 4,4 & 4,3 & 4,0 & 4,0 & 4,1 & 4,2 \\
\hline AVASICION & 3 & 4 & 5 & 3 & 1 & 3 & 5 & 5 & 7 & 2 & 3 & 6 & 6 & 5 & 4 \\
\hline
\end{tabular}

De los alumnos encuestados para poco más de dos tercios de los estudiantes fue su primera experiencia de trabajo con pósteres, y más del $80 \%$ de los encuestados calificó esta experiencia como positiva o muy positiva. Al intentar establecer si la experiencia anterior con la metodología de pósteres podría tener un 
efecto en la valoración de los y las estudiantes de la experiencia, se llevó a cabo una contrastación basada en la prueba $U$ de Mann-Whitney (Altman y Bland, 2009), que no arrojó diferencias significativas (U=115,5, $\mathrm{p}=.538$ ) entre el grupo de estudiantes que tenían o no experiencia previa con los pósteres.

Respecto de los aspectos positivos reportados, ellos se agruparon en: (1) Identificación de lo más importante, sintetizando contenidos, esto es, desarrollar poder de síntesis, destacando lo relevante y marginando lo secundario o irrelevante; (2) Desarrollo de un trabajo grupal, en forma estructurada, constante y dinámica, lo que implica aprender a distribuir el trabajo entre los integrantes del grupo, a compartir el conocimiento, a criticar y recibir críticas, a analizar distintas opiniones; (3) Contribución a organizar la información y clasificar ideas para optimizar el uso del espacio disponible, forzando la necesidad de ordenar y centrarse en lo esencial; (4) Exposición y elaboración de pósteres en forma didáctica y creativa apoyándose en recursos gráficos motivadores; y (5) Trabajo con empresas, compartiendo e interactuando con nuevas personas para abordar problemas reales.

Respecto de los aspectos a mejorar reportados, ellos se agruparon en: (1) Desinformación de los estudiantes respecto de lo que es un póster, sus características centrales, y su elaboración; (2) Evaluación de los pósteres por parte de los estudiantes, percibida como no objetiva a pesar de la existencia de una tabla de rúbricas a considerar; (3) Organización del trabajo grupal para gestionar apropiadamente el tiempo disponible, lo que demanda un espíritu de cooperación entre los integrantes de los grupos de trabajo; y (4) Conformación aleatoria de los grupos de trabajo, constituidos cada uno por 3 estudiantes de forma tal que un estudiante asuma un rol de dirección, otro de creación, y otro de análisis crítico.

Estas respuestas invitan a emprender acciones orientadas a: a) Informar respecto de los objetivos y las características de los pósteres, junto con dar a conocer modelos de pósteres; b) Entregar y explicar exhaustivamente pautas (rúbricas) de evaluación de pósteres que oriente más claramente la evaluación por parte de los estudiantes; y c) Reforzar la importancia del trabajo grupal, la interdependencia que se genera y la responsabilidad que asume cada integrante del grupo al efectuar la evaluación de sus pares y para alcanzar un resultado final exitoso.

En relación a las competencias declaradas en el programa de formación fundamental (PFF) contemplado en todos los planes de formación de las carreras que imparte la Universidad de Talca, los estudiantes sienten que la experiencia de trabajo con pósteres ha contribuido a desarrollar más fuertemente las siguientes competencias:

(1) Entender una situación, desagregándola en sus partes o identificando sus implicaciones paso a paso; organizando las partes de un problema o situación de forma sistemática, realizar comparaciones entre diferentes elementos o aspectos, y establecer prioridades de forma racional (44\%)

(2) Tener una mirada crítica del propio trabajo, mostrar inquietud y curiosidad constante por saber más sobre cosas, hechos y personas (32\%)

(3) Comprender, analizar y relacionar información de diferentes disciplinas del conocimiento y relacionarlas con las aplicaciones de la propia especialidad; utilizar y adaptar los conceptos teóricos, o adquiridos con la experiencia, a situaciones diferentes; integrar ideas, datos y alcances, presentándolos en forma clara y útil; diseñar nuevos sistemas o procedimientos para la especialidad, aplicando información y conocimientos científicos de otras disciplinas (32\%)

(4) Adaptarse y trabajar eficazmente en distintas y variadas situaciones y con personas y grupos diversos; entender y valorar posturas distintas o puntos de vista encontrados, o bien adaptar el propio enfoque a medida que la situación lo requiera; aceptar sin problemas los cambios en el grupo o en las responsabilidades (29\%);

(5) Desear realizar bien el trabajo o sobrepasar un estándar, que puede ser el propio rendimiento en el pasado, una medida objetiva, superar a otros, metas personales que uno mismo se ha marcado o cosas que nadie ha realizado; procurar un logro único y excepcional (29\%);

(6) Identificar un problema, obstáculo u oportunidad y llevar a cabo acciones para dar respuesta a ellos; predisposición a actuar en forma inmediata y no limitarse a pensar en lo que hay que hacer en el futuro; buscar formas más eficientes de hacer el trabajo, de perfeccionar las actividades normales en que se está involucrado; proponer soluciones o distintas formas para ejecutar labores normales o nuevas labores (26\%)

Respecto del aporte al aprendizaje de los estudiantes por parte de los distintos instrumentos de evaluación aplicados en el módulo, la exposición de pósteres ocupó la segunda posición, ocupando la primera posición 
las pruebas. Estos resultados revelan que la exposición de pósteres es visualizada como una interesante, atractiva y motivadora alternativa de aprendizaje para: a) Trabajar en equipo para capturar un problema real; b) Analizar críticamente una problemática en particular con miras a su comprensión y descomposición; y c) Organizar y presentar una solución a un problema real en un formato sintético y expresivo que recoja lo relevante en desmedro de lo secundario.

Tomando en consideración el valor que los y las estudiantes asignaron a cada tipo de evaluación en la construcción de su aprendizaje, aplicando la prueba Phi (Dytham, 2011), la Tabla 3 muestra que no existe una diferencia significativa entre quienes enfrentaban el trabajo de póster por primera vez y quienes ya tenían experiencia con el formato evaluativo.

Tabla 3: Valoraciones de cada formato evaluativo como relevante en el aprendizaje, según estudiantes con y sin experiencia previa con pósteres. El estadístico no ha podido ser calculado porque los valores son constantes

\begin{tabular}{|l|l|c|c|c|c|}
\hline \multirow{2}{*}{} & \multicolumn{4}{|c|}{$\begin{array}{c}\text { Estudiantes que jerarquizaron cada forma de evaluación } \\
\text { como más relevante en su aprendizaje }\end{array}$} \\
\cline { 3 - 6 } & & $\begin{array}{c}\text { En primera } \\
\text { opción }\end{array}$ & $\begin{array}{c}\text { En segunda } \\
\text { opción }\end{array}$ & $\begin{array}{c}\text { En tercera } \\
\text { opción }\end{array}$ & $\begin{array}{c}\text { Sig. Prueba } \\
\text { Phi }\end{array}$ \\
\hline \multirow{2}{*}{ PRUEBAS } & Sin experiencia & 4 & 1 & 4 & .159 \\
\cline { 2 - 6 } & Con experiencia & 13 & 6 & 3 & \\
\hline \multirow{2}{*}{ CONTROLES } & Sin experiencia & 0 & 4 & 1 & .609 \\
\cline { 2 - 6 } & Con experiencia & 1 & 9 & 6 & \\
\hline \multirow{2}{*}{ FICHAS } & Sin experiencia & 1 & 1 & 1 & $*$ \\
\cline { 2 - 6 } & Con experiencia & 1 & 1 & 1 & \\
\hline \multirow{2}{*}{ PRESENTACIONES } & Sin experiencia & 4 & 1 & 1 & .091 \\
\cline { 2 - 6 } & Con experiencia & 0 & 1 & 3 & \\
\hline \multirow{2}{*}{ PÓSTERES } & Sin experiencia & 1 & 3 & 3 & .683 \\
\cline { 2 - 6 } & Con experiencia & 5 & 6 & 5 & \\
\hline \multirow{2}{*}{ INFORME FINAL } & Sin experiencia & 1 & 1 & 1 & .829 \\
\cline { 2 - 6 } & Con experiencia & 2 & 1 & 3 & \\
\hline \multirow{2}{*}{ INF. EJECUTIVOS } & Sin experiencia & 0 & 0 & 1 & $*$ \\
\cline { 2 - 6 } & Con experiencia & 0 & 0 & 0 & \\
\hline
\end{tabular}

\section{CONCLUSIONES}

Los resultados de la experiencia a la fecha son altamente estimulantes en términos de motivación, de desarrollo de las competencias de comunicación tanto orales como de expresión gráfica, y particularmente de trabajo en equipo que la construcción de un póster supone, y en el que los integrantes del equipo asumen distintos roles según sus respectivas potencialidades.

El hecho que la construcción de un póster sea una actividad formativa y evaluativa no convencional implica una serie de desafíos, especialmente focalizados en la necesidad de estructurar y guiar la actividad de manera que no se induzca una sensación de ansiedad por desconocimiento de los pasos a seguir. Es imprescindible dedicar tutorías específicas a conocer el formato y sus requerimientos, adelantar la dinámica de la o las sesiones de exposición y retroalimentación de sus avances y realizar aproximaciones sucesivas con retroalimentación estructurada para asegurar el mejor desempeño posible de los estudiantes en esta actividad.

Los estudiantes de educación superior en Chile no están familiarizados con la idea de póster profesional, por lo que al ser presentados con la actividad, tienden a imaginar pósteres de la línea de manualidades o de educación básica o media, lo que puede afectar su motivación a comprometerse de lleno en la actividad propuesta. El presentar a los estudiantes con fotografías, modelos o ejemplos concretos de presentación profesional por medio de pósteres ayuda a que comprendan la utilidad de manejar el formato en su vida estudiantil y luego profesional.

Si la actividad incluye una forma de evaluación diferente de la heteroevaluación del docente, también es muy necesario que el estudiante sepa cuál es su función en la evaluación de su trabajo y posiblemente el de 
sus compañeros. Ya que la actividad en sí misma implica desempeños relativamente novedosos para los estudiantes, es de vital importancia generar una rúbrica clara para las dimensiones a ser evaluadas tanto en el proceso como en el producto final y también generar espacios de desarrollo de otras habilidades que son necesarias para el buen desarrollo de la actividad, como la diagramación del material a presentar, por ejemplo. En el intento de despejar el impacto de la metodología de póster como formato evaluativo novedoso para los y las estudiantes, se examinaron posibles diferencias de valoración general y por formato evaluativo dependiendo si los o las estudiantes habían tenido experiencia con pósteres anteriormente. El resultado de tales pruebas no arroja diferencias significativas de valoración entre las categorías de estudiantes, implicando que la valoración que los y las estudiantes hacen de las metodologías evaluativas no está significativamente asociada a la experiencia con el formato.

Si se aspira que los avances de preparación de los pósteres contribuyan al progreso en el aprendizaje de los alumnos, y que tales avances estén alineados con el desarrollo de la correspondiente asignatura, la implementación de esta actividad y su evaluación requieren de una dedicación especial del tiempo de cátedra, y por tanto, contemplarse en los creditajes pertinentes.

A medida que los estudiantes progresan en la actividad, sus fortalezas y debilidades para el desarrollo de ésta se hacen evidentes y generalmente se focalizan en falta de conocimiento del formato. Esta incertidumbre les dificultaría escoger adecuadamente el material a presentar, tomar buenas decisiones en el manejo del espacio de presentación, o errar en el nivel de lenguaje escogido en los elementos a incorporar. Es por eso que las aproximaciones estructuradas y la retroalimentación son relevantes, para poder eliminar los errores causados por el desconocimiento del ritmo y el formato de trabajo, que podrían interferir con la capacidad de los estudiantes de demostrar las habilidades trabajadas en el módulo.

La o las instancias de presentación deben ser muy claras en los roles, tiempos y actividades a realizar, ya que la convergencia de estudiantes en un espacio que podría ser reducido, y su necesidad de circular, podrían crear un problema logístico para la evaluación y comunicación entre los asistentes. Es muy importante intencionar la forma y el contenido de la interacción entre pares, para que la retroalimentación hecha a cada póster sea relevante, tanto a nivel formativo como de modelado de habilidades profesionales.

En resumen, las limitaciones para llevar a cabo la propuesta pedagógica de trabajo con pósteres se agrupan en 2 categorías: la capacidad del estudiante para incorporar este formato dentro de sus actividades de aprendizaje, y la capacidad del profesor para reorganizar su trabajo docente. Dentro de la primera se incluyen las eventuales dificultades para trabajar en grupos conformados aleatoriamente y para evaluar el trabajo de otros grupos; en la segunda categoría los obstáculos a enfrentar se relacionan con la disponibilidad de tiempo para organizar y orientar el trabajo de los estudiantes en un formato al cual no están habituados.

La incorporación de profesionales o académicos de otras áreas también es una opción muy interesante a integrar en el momento de la presentación. La interacción multidisciplinar exige de los estudiantes la capacidad de transponer un lenguaje interno a uno de difusión sin perder la posición profesional, además de la capacidad de integrar conocimientos al responder una pregunta o comentario que no necesariamente se limita a los confines del módulo presente, sino a una labor profesional más integrada.

Finalmente, la calificación de tal esfuerzo también plantea un desafío en la integración de distintas evidencias que podrían haber sido evaluadas: progreso del trabajo en equipo, calidad de los aportes individuales, calidad de la interacción en la presentación, diferencias de calidad entre distintos puntos de control formativos, evaluación de pares en el producto final, autoevaluación y, evaluación de profesionales externos o clientes y/o la evaluación del docente. La fórmula escogida debe ser plenamente conocida, comprendida y lógica para los estudiantes, sin descartar su participación en el establecimiento de los criterios a utilizar.

\section{REFERENCIAS}

Altman, D. y Bland, J.M., Parametric v non-parametric methods for data analysis. BMJ, 338, (2009).

Araya, I., La Formación Dual y su fundamentación curricular. Revista Educación, 32(1), 45-61. Costa Rica. (2008).

Aristimuño A., Las competencias en la educación superior: ¿demonio u oportunidad?. Departamento de Educación, Universidad Católica de Uruguay, 2004. http://www.cedus.cl/files/competencia_aristumuño.pdf. Acceso: 24 agosto (2011). 
Bezanilla, M., El proyecto Tuning y las competencias específicas. Seminario Internacional "Orientaciones pedagógicas para la convergencia europea de Educación Superior". Universidad de Deusto, julio 2003. http://www.ucm.es/centros/cont/descargas/documento4012.pdf. Acceso: 15 abril (2011).

Biggs, J., Teaching for Quality Learning at University. En Assessing for learning quality: II. Practice (pp. 165203). University Buckingham, UK: SRHE and Open University Press (1999).

Bracher, L., Cantrell, J., y Wilkie, K., The process of poster presentation: a valuable learning experience. Medical Teacher, 20(6), 552-557. (1998).

Brunner, J.J., Educación Superior en Iberoamerica: Informe 2007. CINDA. Santiago-Chile. (2007).

Brunner, J.J., El proceso de Bolonia en el horizonte latinoamericano: límites y posibilidades. Revista de Educación del Ministerio de Educación, España, número extraordinario 2008, 119-145. (2008).

Cates, C.L. y Langford, D.R. Documenting Communication and Thinking Skills Trough Co-op Student Reports. Journal of Cooperative Education. 34(3). 7-17. (1999).

Dytham, C., Choosing and using statistics: A biologist's guide. Oxford: Wiley-Blackwell. (2011).

Dochy, F., Segers, M., y Sluijsmans, D., The use of self-, peer and co-assessment in higher education: a review. Studies in Higher education. 24(3), 331-350. (1999).

Faúndez F., Gutiérrez A. y Ponce M., Desarrollo institucional del currículo basado en competencias: el caso de la Universidad de Talca. Documento de Trabajo-Universidad de Talca. (2009).

González, J., Wagenaar, P. y Beneitone, P., Tuning-América Latina: un proyecto de las universidades. Revista Iberoamericana de Educación, 35, 151-164. (2004).

Greinert, W.D., Los "sistemas" europeos de formación profesional: algunas reflexiones sobre el contexto teórico de su evolución histórica. Revista Europea Thessaloniki, 2(32), 18-26. (2004).

Handron, D., Poster Presentations A Tool for Evaluating Nursing Students. Nurse Educator, 19(1), 17. (1994).

Johnson D.W., Johnson R.T. y Smith K.A., Cooperative Learning Returns to College: What Evidence Is There That It Works?. Change: The Magazine of Higher Learning, 30(4), 26-35. (1998).

Le Boterf G., Ingénierie et évaluation des competences. Paris: Les Editions d'Organization. 2001.

Lester, S. y Costley, C., Work-based learning at higher education level: Value, practice and critique. Studies in Higher Education, 35, 561-575. (2010).

Moneyham, L., Ura, D., Ellwood, S., y Bruno, B., The poster presentation as an educational tool. Nurse Educator, 21(4), 45-47. (1996).

Moore, L. W., Augspurger, P., King, M. O., y Proffitt, C., Insights on the poster preparation and presentation process. Applied Nursing Research, 14(2), 100-104. (2001).

Orsmond, P., Merry, S., \& Reilingi, K., The Use of Student Derived Marking Criteria in Peer and Selfassessment. Assessment \& Evaluation in Higher Education, 25(1), 23-38. (2000).

Prieto, J.P., Pey R., Durán F., Villarzú A., Larraín A.M., Detmer A., Vargas G., Stevenson N., Del Valle, R., Torres, R., Bendersky S., Heyl V. y Concha X., Articulación y movilidad: apuntando a la eficiencia del sistema. PROPUESTAS PARA LA EDUCACIÓN SUPERIOR Foro Aequalis y las transformaciones necesarias. Santiago-Chile. (2011).

Schmal, R. y Ruiz-Tagle, A., Un modelo para la gestión de una escuela universitaria orientada a la formación basada en competencias. Cuadernos de Administración, 22(39). 287-305. (2009).

Schmal, R., Ruiz-Tagle, A. y Cerpa, N., Una experiencia docente de vinculación universidad-empresa. Congreso Nacional de Educación en Ingeniería. (2009).

http://utalca.academia.edu/NarcisoCerpa/Papers/658338/Una_experiencia_docente_de_vinculacion_Univers idad-Empresa. Acceso: 23 marzo (2012). 
Schmal, R. y Ruiz-Tagle, A., Un nuevo profesional para la gestión organizacional. Revista del Instituto Internacional de Costos. Edición Especial XII Congreso, abril 2012. 87-96. (2012).

Schmal, R., Reflexiones en torno a un programa para la formación de competencias transversales en ingeniería. Revista Ciencia, Docencia y Tecnología, año XXIII n 44. 239-262. (2012).

UTalca, Modelo Educativo: Universidad de Talca. Vicerrectoría Docencia Pregrado. Talca-Chile. 31 p. (2012).

Venables, A., Tan, G. y Bellucci, E., The impact of learning in the workplace policy on differing ICT degrees, in WACE 2009 : Proceedings of the WACE 16th World Conference on Cooperative Education and Work Integrated Learning, World Association for Cooperative Education, Boston, Mass., 1-6. (2009). http://hdl.handle.net/10536/DRO/DU:30032777. Acceso: 10 junio (2012). 